\title{
BERATUNG
}

\section{Führungskräfte sind einsam}

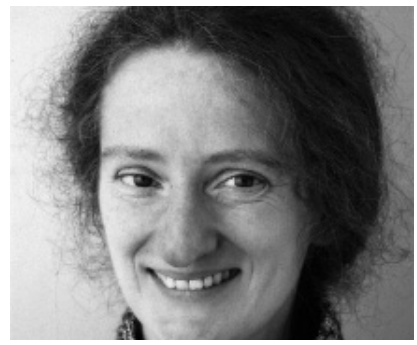

VON EVA DOUMA

Dr. Eva Douma ist Sozial- und Verwaltungswissenschaftlerin. In Frankfurt am Main arbeitet sie seit 15 Jahren als Business-Coach und Beraterin für die Sozialwirtschaft. Ihre Arbeitsschwerpunkte sind die Personal- und Organisationsentwicklung.

Internet http://www.douma.de

\author{
Wer an der Spitze steht, steht häufig allein. Damit \\ Führungskräfte besseren Halt und Orientierung finden, \\ sollten sie sich angemessene Unterstützung \\ organisieren.
}

Ein unvoreingenommener Blick von außen kann helfen, für kritische Fragestellungen adäquate Lösungen zu entwickeln. Die Außenperspektive erweitert den Horizont, lenkt den Blick auf andere Gesichtspunkte und kann einer Betriebsblindheit entgegenwirken. Das eigene Verhalten wird reflektiert und kann modifiziert werden. Zwei Formen der Unterstützung werden nachfolgend vorgestellt. Welche Hilfe für wen infrage kommt, hängt von den persönlichen Anforderungen und von den zu klärenden Fragen ab.

\section{Kollegiale Beratung}

heißt, dass sich Menschen in vergleichbaren Situationen zusammenfinden und sich gegenseitig beraten. Die konkrete Fallbearbeitung steht im Zentrum: »Meine Situation ist die folgende, welche Erfahrungen haben die anderen in ähnlichen Konstellationen gemacht? Was unternahmen sie mit welchem Ergebnis? «

Dies sind Fragestellungen, die mit einer kollegialen Beratung gut bearbeitet werden können. Damit diese Methode funktioniert, bedarf es einer vertrauensvollen, gleichberechtigten Beziehung der Beteiligten zueinander. Vier bis maximal acht Teilnehmende bilden gruppendynamisch eine günstige Gruppengröße. Kollegiale Beratungsgruppen bilden sich innerhalb eines Unternehmens oder gezielt außerhalb des Betriebes. Entscheidend ist, dass die Beteiligten konfliktfrei und offen miteinander umgehen und nicht in Konkurrenz zueinander stehen.

Damit vorschnelle Bewertungen unterbleiben und eigene Lösungen gefunden werden, sollte die kollegiale Beratung durch ein formalisiertes Verfahren strukturiert werden. Regelmäßige Treffen und ein ritualisierter Ablauf unterstützen dies. Eine kollegiale Beratung kann zehn Phasen durchlaufen:

1. Phase Rollenbesetzung: Die Gruppe einigt sich auf einen Moderator, der die weiteren Phasen strukturiert und dokumentiert. »Fallgeber « kann jeder sein, der für ein Schlüsselthema oder eine schwierige Situation neue Perspektiven oder Lösungsideen sucht. Die übrigen Gruppenmitglieder bilden das Beraterteam.

2. Phase Fallerzählung: Der Fallgeber berichtet, worum es geht. Das Beratungsteam hält sich zurück. Verständnisfragen werden am Ende geklärt.

3. Phase Schlüsselfrage: Der Fallgeber formuliert seine Schlüsselfrage und wird hierbei durch die Moderation unterstützt. Eine hilfreiche Schlüsselfrage umreißt klar, was sich der Fallgeber als Ziel der kollegialen Beratung wünscht.

4. Phase Auswahl der Beratungsmethode: Der Moderator leitet die Auswahl einer Beratungsmethode, die der Bearbeitung der Schlüsselfrage dient.

5. Phase Fragen zum Sachverhalt: Diese Phase dient der (erneuten) Klärung von Verständnis- und Informationsfragen - diesmal unter dem Fokus der Schlüsselfrage. Es werden zu diesem Zeitpunkt noch keine Probleminterpretationen durch das Beraterteam geliefert. 
Die ersten fünf Phasen sollten jeweils maximal fünf Minuten beanspruchen.

6. Phase Hypothesenbildung: In einer zehnminütigen Phase schweigt der Fallgeber. Das Beratungsteam formuliert Vermutungen, Hypothesen und Eindrücke. Es werden in dieser Phase keine Lösungen entwickelt.

7. Phase Stellungnahme: Maximal fünf Minuten hat der Fallgeber Gelegenheit, die Hypothesen des Beratungsteams durch weitere Informationen zu korrigieren. Das Beratungsteam hört zu und korrigiert gegebenenfalls seine $\mathrm{Hy}$ pothesen aus der letzten Phase. Es wird nicht diskutiert.

8. Phase Lösungsvorschläge: Mit Hilfe der gewählten Beratungsmethode entwickelt das Beratungsteam zehn Minuten lang Lösungsvorschläge zur Schlüsselfrage. Der Fallgeber hört in dieser Phase zu und lässt die Ideen der Beratenden auf sich wirken.

9. Phase Entscheidungsphase: Der Fallgeber nimmt zu den aus seiner Sicht hilfreichen Anregungen Stellung. In dieser etwa zehnminütigen Phase teilt der Fallgeber mit und begründet, welche Hypothesen er sich zu eigen macht und welche Vorschläge er umsetzen möchte. Das Beratungsteam hört zu. Es wird nicht diskutiert.

10. Phase Auswertung: In der Abschlussrunde wird das Verfahren ausgewertet. Rund fünf Minuten tauschen sich die Beteiligten über die erfolgte Beratung aus.

Der ganze Beratungszyklus sollte insgesamt nicht länger als eine Stunde dauern. Ist er beendet, werden die Rollen gegebenenfalls neu verteilt.

\section{Business-Coaching}

hat zum Ziel, für komplexe Entscheidungs- und Konfliktsituationen am Arbeitsplatz Lösungen zu entwickeln. Der Schwerpunkt liegt auf der Entwicklung beruflicher Handlungsoptionen. Der gezielte Blick von außen verschafft einen Perspektivwechsel, es werden Handlungsalternativen entwickelt und Erfahrungen reflektiert.

Anlass für ein Business-Coaching können Konflikte mit Vorgesetzten oder Mitarbeiterinnen und Mitarbeitern, Kommunikationsprobleme, ein organisatorischer Wandel oder artverwandte
Fragestellungen sein. Das Gespräch findet zwischen der Führungskraft und einem externen Beratenden unter vier Augen statt. Persönliche Fragen können leichter und intensiver als in einer Gruppe angesprochen werden.

Business-Coaching heißt nicht, dass der Beratende die Entscheidungen anstelle der Führungskraft trifft. Statt dessen ist es die Aufgabe der Coaches, die Führungskraft offensiv dabei zu unterstützen, für eine (in der Regel schwierige) Situation eine adäquate Lösung zu finden. Ein guter Coach hilft, klarer zu sehen und auf dieser Basis eine Entscheidung zu treffen.

Ein Business-Coaching findet bewusst nicht im beruflichen Umfeld, sondern auf »neutralem « Terrain statt. Hier kann sich die Führungskraft ungestört und entspannt über ihre professionelle Situation Klarheit verschaffen. In Abhängigkeit zur Aufgabenstellung können mehrere, durchaus mehrstündige, Sitzungen durchgeführt werden. Vorab sollten Fragestellung und Zeitplan definiert werden. Tauchen neue Fragestellungen auf, steht einem weiteren Business-Coaching nichts im Wege - auf der Grundlage einer neuen Vereinbarung. Gewöhnlich läuft ein Business-Coaching in sechs Phasen ab:

1. Phase Bedarf und Aufgabenstellung formulieren: Erwartungen der Führungskraft an das Coaching werden formuliert. Es wird geklärt, inwieweit diese erfüllbar sind. Wenn Coach und zu Coachende von der Wirksamkeit einer Zusammenarbeit überzeugt sind, werden eine klare Aufgabenstellung und ein Zeitplan vereinbart.

2. Phase Situationsanalyse: Der zu Coachende beschreibt die berufliche Situation und die sich ergebenden Schwierigkeiten. Eine Wertung durch den Beratenden findet in dieser Phase nicht statt.

3. Phase Konkrete Ziele des Coachings definieren: Das Ergebnis, das am Ende des Coaching-Prozesses stehen soll, wird beschrieben.

4. Phase Entwicklung von Handlungsalternativen: Der Coach unterstützt die Führungskraft, neue Sichtweisen auf das eigene Handeln zu entwickeln. Die Wirkung des eigenen Verhaltens wird reflektiert. Alternative Verhaltensweisen für konkrete berufliche Situationen werden entwickelt und in Übungen trainiert. Geklärt wird, was wann wie durch wen zu tun ist. Im Zentrum steht die Steigerung der Handlungs- und Lösungskompetenz.

5. Phase Überprüfung der Zielerreichung: In dieser Prozessphase wird geprüft, inwieweit die erarbeiteten Handlungsalternativen zum eingangs formulierten Ziel führen.

6. Phase Evaluation des Coaching-Prozesses: Unabhängig vom Ergebnis der Zielerreichung wird die Zufriedenheit des zu Coachenden abgefragt. Die Nachhaltigkeit der Verhaltensänderungen wird überprüft.

\section{Die richtige Beratung finden}

Unabhängig davon, für welche Methode Sie sich entscheiden: Damit eine Beratung durch Kollegen und Kolleginnen oder durch einen Coach funktioniert, sollten Sie der Suche nach der richtigen Beratung die notwendige Aufmerksamkeit widmen - sowohl bezüglich der Form als auch der Person(en).

- Klären Sie vorab, welches Problem Sie lösen wollen und welche Unterstützung Sie dafür benötigen. Je mehr Sie wissen, was Sie wollen, desto größer ist die Chance, dass Sie das bekommen, was Sie benötigen.

- Klären Sie, was zur Disposition steht und was nicht.

- Klären Sie, inwieweit der Berater sich mit Ihren spezifischen Fragestellungen auseinandersetzen kann und will.

- Ihr Berater muss nicht sein wie Sie. Eine zu große Ähnlichkeit widerspricht dem Ziel der Außenperspektive. Aber Konflikte, die im Beratungsprozess entstehen (können), müssen thematisierbar und lösbar sein.

- Vereinbaren Sie eine klare Aufgabenstellung und ein entsprechendes Beratungsdeputat. Verändert sich im Beratungsprozess die Fragestellung, treffen Sie eine neue Vereinbarung.

- Eine Dauerberatung löst keine Probleme. So sollte jede Beratung nicht nur einen klar definierten Anfang, sondern auch ein Ende haben.

\section{Literaturtipp}

Bernd Schmid, Thorsten Veith, Ingeborg Weidner: Einführung in die kollegiale Beratung. Carl Auer Verlag, Heidelberg 2010. 126 Seiten.

12,95 Euro. ISBN 978-3-89670-731-4. 